Check for updates

The BMJ

Cite this as: $B M J 2022 ; 376: 0265$ http://dx.doi.org/10.1136/bmj.0265 Published: 01 February 2022

\title{
Covid-19 patients discharged from hospital have "substantially higher risk" of adverse outcomes and need monitoring
}

\section{Ingrid Torjesen}

Patients who leave hospital after a stay for covid-19 are far more likely to need to be readmitted or to die than the general population, shows a UK study. ${ }^{1}$ The researchers said their findings emphasised that patients discharged after covid-19 were likely to pose a "substantial extra burden on healthcare."

The findings support those of a study published in The BMJ in March 2021 that found that patients of all ages discharged after hospital treatment for covid-19 had higher than expected rates of multiorgan dysfunction. ${ }^{2}$

In the new study, researchers used linked primary care and hospital data in England to evaluate the risks of hospital admission and death among 24673 patients who were treated in hospital for covid who survived for at least a week after discharge between February and December 2020. They were compared with 123362 matched controls. Outcomes among the discharged patients were also compared with outcomes in 16058 people who had been discharged after hospital treatment for influenza between 2017 and 2019.

The findings, reported in PLoS Medicine, showed that during a follow-up period of up to 315 days the covid patients were more than twice as likely to need readmission or to die as the general population (fully adjusted hazard ratio 2.22 ( $95 \%$ confidence interval 2.14 to $2.30, \mathrm{P}<0.001)$ ) but slightly less likely than the patients who had been admitted to hospital for flu (HR 0.95, 0.91 to 0.98, $\mathrm{P}=0.004$ ).

A total of 7439 of the 24673 patients admitted for covid-19 died during follow-up, meaning they more than four times as likely (HR 4.82 (4.48 to 5.19, $\mathrm{P}<0.001)$ to die from any cause than the general population controls and also more likely than the flu patients (HR 1.74 (1.61 to 1.88), $\mathrm{P}<0.001$ ). Risks for all disease specific hospital admissions and deaths were greater among former covid patients than the control group but similar to or lower than those among the flu patients, except for respiratory and mental health outcomes.

Patients admitted to hospital for covid-19 were also more likely than those admitted for flu to be readmitted or to die because of their initial infection or other lower respiratory tract infections (HR 1.37 (1.22 to 1.54, $\mathrm{P}<0.001)$ ). They were also more likely to experience a mental health or cognitive related admission or death (HR 1.37 (1.02 to 1.84, $\mathrm{P}=0.039$ )). In particular, former covid patients with pre-existing dementia had more than double the risk of hospital readmission for dementia or of death (age and sex adjusted HR 2.47 (1.37 to 4.44, $\mathrm{P}=0.002$ )) than the former flu patients.
"These findings suggest a need for services to support and closely monitor people following discharge from hospital with covid-19-for example, through more frequent/active follow-up in primary care in the weeks and months following a hospitalisation," concluded the researchers. hospitalisation and death after COVID-19 hospitalisation in England: A cohort study using linked primary care, secondary care, and death registration data in the OpenSAFELY platform. PLOS Med 2022;19:e1003871. doi: 10.1371/journal.pmed.1003871 pmid: 35077449

2 Ayoubkhani D, Khunti K, Nafilyan V, etal. Post-covid syndrome in individuals admitted to hospital with covid-19: retrospective cohort study. BMJ 2021;372:n693. doi: 10.1136/bmj.n693 pmid: 33789877

This article is made freely available for personal use in accordance with BMJ's website terms and conditions for the duration of the covid-19 pandemic or until otherwise determined by BMJ. You may download and print the article for any lawful, non-commercial purpose (including text and data mining) provided that all copyright notices and trade marks are retained. 